\title{
MEASURING CLASSIFICATION ACCURACIES USING IMPROVED THERMAL INFRARED DATA
}

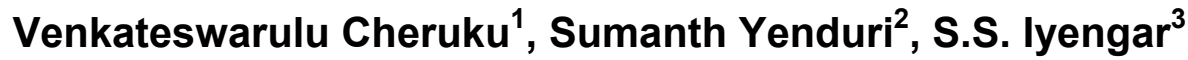

(1) Software Engineer, Infosys Technologies Ltd, Hyderabad, India, Venkateswarlu_C@infosys.com

(2) Ph.D. Student, Louisiana State University, Baton Rouge, USA, sumanth@bit.csc.lsu.edu

(3) Professor, Louisiana State University, Baton Rouge, USA, iyengar@bit.csc.lsu.edu

\begin{abstract}
Image classification is one of the major aspects in digital image analysis of remotely sensed data. In this paper, we present the effects on classification accuracy if improved thermal data are used instead of raw thermal data. We use two methods, Artificial Neural Networks (ANN) and Maximum Likelihood Approach (MLH) to demonstrate our purpose. Using each method different combinations of raw and improved data are tested to classify in order to compare the accuracies. As a final note, the findings are discussed.
\end{abstract}

Keywords: Artificial Neural Networks, Classification Accuracy, Maximum Likelihood Approach, Thermal Infrared Data

\section{REVIEW}

The interpretation of the Thermal Infra Red (TIR) imageries has been effectively used in geological mapping. Stephen and Venugopal [1] conducted a study on the utility of Landsat TM band 6 thermal infrared data (10.4 to $12.5 \mathrm{~mm}$ ) for urban feature analysis. They used several combinations of Landsat $\mathrm{TM}, 7$ bands data to determine the relative importance of the thermal band as a component of an urban land use classification. To calculate the inter-class separability for parametric classification they used the descriptive statistics like spectral response means, standard deviation and correlation etc. Comparisons were made on the classified images, which resulted from the supervised layered classification when the thermal data are used and not used in the analysis. The results demonstrated that the information content of thermal data were valuable for urban area analysis. Topographic effects dominate daytime images whereas on nighttime images topographic features are eliminated and geologic features are emphasized [2]. TIR data contain additional valuable information of emittance of materials and are very much useful in land surface classification.

Price [3] and Ormsbay [4] found classification results are quite acceptable when TIR data are used along with visible and near infrared data. TIR data can also be used to improve the accuracy of classification when used along with other highresolution data. They conducted an investigation on the contribution of thermal data of Landsat-3 Multi Spectral Scanner (MSS) in conjunction with visible and near infrared data. They noticed that classification using Landsat 1 and 2 images some times might not be unique. Since thermal-IR channel is relatively un-correlated with the others, one may anticipate some impact on the classification procedure. However, the numerous physical processes governing thermal radiation lead to a dependence on surface slope, altitude, and surface energy-balance effects such as ground heat flux, atmospheric heating, and surface evaporation. Because of the differing physical properties of the thermal data, their use in classification is subject to ambiguities and dependencies on other factors that make analysis prone to error.

Chavez et al. [11] carried out a comparative study on three different methods to merge multiresolution and multispectral data (Landsat TM with SPOT PAN). Landsat TM data are digitally enlarged by a factor of three in both directions to generate a pixel size similar to PAN data in order to register both the images geometrically. The enlarged TM data are smoothed by $3 \mathrm{X} 3$ low pass filter to eliminate the blockiness introduced by the $3 \mathrm{X}$ digital enlargement of Image. The three methods, they used for merging data are i) Hue Intensity Transformation (HIS), ii) Principle Component Transformation (PCT), and iii) High Pass Filter (HPF). From the comparison of results, they concluded that the results generated by HPF method are less distorted than the results 
generated by either HIS or PCT. The PCT method distorted the spectral characteristics of the data less than the HIS method. One more advantage of using HPF method is that the same PAN spatial filter output can be applied to all the bands, or any subset of the bands, and any low frequency noise problems existing in the higher spatial resolution image will be automatically removed. They also suggested the best kernel size was approximately twice the size of the ratio of the different spatial resolution.

Artificial Neural networks were proved to be the better over the existing probabilistic methods for classification of remotely sensed data. Hence, ANNs were used for accurate land use/land cover classification. In classification by neural network approach three types of layers were used by Foody and Arora [8]. Heermann and Khazenie varied the number of hidden nodes in the neural network to result in more rapid learning. Back propagation networks were slow to train, but were fast in the classification stage. The network was also compact, which would allow classification information to be distributed as network rather than another image channel [5]. Bischof et al integrated textural information and used them with neural networks classifiers to increase the accuracy of classification [6].

Factors affecting the classification accuracy of neural network method were investigated by Foody and Arora [8]. They are network architecture, training set size, discriminating variables and testing data characteristics. It was concluded that in general, larger, more complex, neural networks would be able to correctly characterize a training set at the behest of adequate generalization. Size of training set for each class must be at least 10-30 times the number of discriminating wavebands. Increase in the training set size results in small increase in classification accuracy, but at the expense of marked increase in processing time. Classification accuracy rises initially with an increase in number of bands used to a point beyond which the addition of data acquired in other spectral bands has no significant effect, or results in reduction in accuracy. Nature of testing set can have significant effect on accuracy; hence the testing set must be representative of the classes. Therefore, test samples should be drawn from across the test site and the sample should be large enough.

\section{INTRODUCTION}

TIR data can also be used to improve the accuracy of classification when used along with other high-resolution data. In the present study, ANN and MLH approaches are used for classification. Classification is carried out using raw and improved thermal data and the results are compared. The spatial resolution of remotely sensed data in the TIR range is very coarse $(120 \mathrm{~m})$ when compared to visible data $(30 \mathrm{~m})$. Improvement of effective spatial resolution is an attempt to derive the details at the sub-pixel level. The ANN method was not only used to improve the spatial resolution to produce improved thermal data but also used in the classification. Thus the thermal data with improved spatial resolution is what we refer to as "improved data". The improved image had better visual interpretation and also preserved the thermal properties. When we enlarged the thermal data to the size of visible data, we could not visualise anything in the thermal data, as they were blocks like. We used ANN for improving the resolution. We also used ANN in Land use/Land cover classification and proved that this method is superior when compared to the traditional maximum likelihood classification. Various applications where our method could be necessary are Improvement of Classification Accuracy, Distinct identification of Land use/Land cover classes, Temperature mapping using improved thermal images, to monitor the human induced changes of the land, ocean and atmosphere, to better understand and predict the Earth's weather events and seasonal changes so as to reduce risks from natural hazards such as wildfires, droughts etc.

We begin with ANN using raw daytime thermal data and multispectral (MS) data for classification. Next, improved daytime thermal data were used along with MS data. Classification was also done using MS data alone. Raw nighttime thermal data were used in classification along with MS data and raw daytime thermal data to notice changes in accuracy. Further, improved daytime and nighttime data were used instead of raw data. Classification was done using Maximum Likelihood Approach for all the above combinations of data. We have tabulated the accuracies for each combination of data (and for each method) individually. Finally we discuss and compare the results obtained. The study was performed on the area around the Thane creek with the suburban of North Mumbai, which consists of thick vegetation, forests, lakes, swamps, barren lands, hills, urban mixed areas etc. Landsat-5 TM, band 6 (TIR) daytime data at $120 \mathrm{~m}$ spatial resolution and band 2, 3, 4 data at $30 \mathrm{~m}$ spatial resolution, acquired on $20^{\text {th }}$ Dec., 1989 and TIR night-time data at $120 \mathrm{~m}$ resolution acquired on $20^{\text {th }}$ Dec 1989 , were available and used for the studies. We did not take any spectral bands. The data had been made available from the satellite with spectral resolution right away. We had only three bands and so our processing was limited to them. Had we used all bands, the results would have been better than now. The bands 2, 3 and 4 data were used in deriving a 
False Colour Composite-FCC and used as a ground truth base map.

For both, daytime and nighttime TIR data, $30 \mathrm{~m}$ spatial resolution data were derived from the $120 \mathrm{~m}$ resolution data, by simple replication to match the resolution of the visible (VIS) and near infrared (NIR) bands. A sub-image of 448 lines X 448 pixels of bands, 2, 3, 4, and band 6 (TIR) image was extracted from the available daytime data of 1536 lines X 1024 pixels. Similarly, a sub-image of 420 lines X 420 pixels was extracted from the original nighttime TIR data of 1024 lines X 1024 pixels. The nighttime data were registered to the daytime data using IDRISI software for further processing. Registration was done by taking few prominent points on both the images and their respective positions were matched.

\section{METHODOLOGY}

The main constraint of the thermal infrared remote sensing is its low (coarse) spatial resolution. It is due to the low energy availability in the thermal infrared region of the electromagnetic spectrum. In order to obtain the accurate ground surface temperatures, the use of multispectral thermal infrared data are preferable. Thermal infrared data contain additional valuable information of emittance of materials and it is very much useful in land surface classification. Thermal infrared data have less correlation with the other reflective band data like visible and near infrared. Thermal infrared data can also be used to improve the accuracy of classification when used along with other highresolution data. Using ANN's we improve the effective spatial resolution of the thermal infrared data. Classification accuracy can be improved if thermal data are used along with visible and near infrared data. Neural networks offer a better classification than the conventional maximum likelihood approach. In the present study ANN and MLH are used for the classification using visible, NIR data, Daytime and Nighttime thermal data.

\subsection{CLASSIFICATION USING ARTIFICIAL NEURAL NETWORKS}

Neural Networks are parallel processing models for information processing systems which consist of a large number of very simple yet highly inter connected processing elements called nodes/units [6]. The Neural Net technique of Back Propagation is used for classifying TIR and MS data. The network was trained using Back Propagation on representative areas of the classes and the resulting Neural Net was used to classify the entire image [5]. The Neural Net uses the training data to adjust the weights connecting the units/nodes until it is able to correctly identify class memberships, which is achieved with the aid of an iterative learning process. In classification by Neural Network approach a three-layered $\mathrm{NN}$ is used. First one is input layer, second one is hidden layer and the third one is output layer. Input layer comprises one node for each input band. Number of hidden nodes in a hidden layer can be calculated using empirical formula [7] given as,

\# Hidden nodes $=(2+$ classesbands $+1 / 2$ classes (bands ${ }^{2}+$ bands $\left.)-3\right) /($ bands + classes $)$

Third, the output layer contains one node for each class [8,9]. All nodes in the network, except input node collect the activation of nodes in the previous layer and set output activation [6].

Collection function is given by,

$$
\operatorname{Net}_{\mathrm{pi}}=\sum \mathrm{w}_{\mathrm{ij}} \mathrm{a}_{\mathrm{pi}}+\text { bias }_{\mathrm{i}}
$$

Where $\mathrm{w}_{\mathrm{ij}}$ represents connection strength for current node $i$ to a node $\mathrm{j}$ in the previous layer $\mathrm{j}, \mathrm{a}_{\mathrm{p} j}$ is the activation of node $j$ for pattern $p$ and $b^{2} s_{j}$ is the node bias. The result of collection function, net $_{p i}$ is passed to the output $\mathrm{a}_{\mathrm{pi}}$, through an activation function, Usually the sigmoid function as given below is used,

$$
\mathrm{a}_{\mathrm{pi}}=1 /\left(1+\mathrm{e}^{-\mathrm{netpi}}\right)
$$

A representative set of input and output pattern is selected. At each input node, the input pattern is presented, the connection weights of the network are adjusted so that activation of nodes more closely matches the desired output pattern [6]. All the patterns are repeatedly presented to the network until the network learns the patterns. Foundation of the Back Propagation of learning algorithm is the nonlinear optimization technique of gradient descent on the sum of squared difference between the activation $\mathrm{O}_{\mathrm{pi}}$, of the nodes in the output layer and the desired output $\mathrm{t}_{\mathrm{pi}}$. The objective is to minimize, therefore $\mathrm{E}$ is given by,

$$
\begin{gathered}
\mathrm{E}=\Sigma \Sigma\left(\mathrm{t}_{\mathrm{pi}}-\mathrm{O}_{\mathrm{pi}}\right)^{2} \\
\mathrm{p} \quad \mathrm{i}
\end{gathered}
$$

where $\mathrm{p}$ are the training patterns and $\mathrm{i}$ is the output node. Weights are updated as,

$$
\Delta \mathrm{w}_{\mathrm{ij}}(\mathrm{n}+1)=\varepsilon\left(\gamma_{\mathrm{pi}} \mathrm{a}_{\mathrm{pj}}\right)+\alpha \Delta \mathrm{w}_{\mathrm{ij}}(\mathrm{n})
$$

where wij is the connection strength from node $\mathrm{i}$ to node $\mathrm{j}$ 
$\gamma p i$ is the node $i$ error for pattern $p$

apj is the activation of node $j$ for pattern $p$

$\varepsilon \quad$ is the learning rate

$\alpha$ is the momentum term

Node error $\gamma p$ i for output node is given

$$
\gamma_{\mathrm{pi}}=\left(\mathrm{t}_{\mathrm{pi}}-\mathrm{a}_{\mathrm{pi}}\right) \mathrm{a}_{\mathrm{pi}}\left(1-\mathrm{a}_{\mathrm{pi}}\right)
$$

At any other arbitrary hidden node the error is adjusted as,

$$
\gamma_{\mathrm{pi}}=\mathrm{a}_{\mathrm{pi}}\left(1-\mathrm{a}_{\mathrm{pi}}\right) \sum \gamma_{\mathrm{pk}} \mathrm{W}_{\mathrm{ki}}
$$

\subsection{CLASSIFICATION USING MAXIMUM LIKELIHOOD APPROACH}

The MLH algorithm can be effectively used for classification of remotely sensed images. In this method, probability of a pixel belonging to each class is calculated and the pixel is assigned to that class with which it has the maximum probability. Gaussian multivariate normal distribution function (Eq 8) is used for probability calculation. If the pixel has less than the user defined threshold probability, the pixel is kept unclassified. $\mu)]$

$$
\mathrm{P}(\mathrm{x} / \mathrm{k})=\left[1 /\left(2 \pi^{\mathrm{d} / 2}|\mathrm{C}|^{1 / 2}\right)\right] * \exp \left[-1 / 2(\mathrm{x}-\mu)^{\mathrm{t}} \mathrm{C}^{-1}(\mathrm{x}-\right.
$$

where $P(x / k)$ is probability of a pixel belonging to class $\mathrm{k}$,

$\mathrm{C}$ is the covariance matrix

$|\mathrm{C}|$ is the Determinant of covariance matrix

$\mathrm{C}^{-1}$ is the inverse of covariance matrix

$\mu$ is the mean vector and $\mathrm{x}$ is the pixel under consideration

\section{TEST RESULTS}

The intent of this paper is to carry out Landuse/Landcover classification and for that we need more than one band. This is because a feature (tone of the feature) might appear differently in different bands. Here we carried out classification using only visible data (Bands 2, 3 and 4), visible data and thermal data (Bands 2, 3, 4 and 6), visible data and improved thermal data (Bands 2, 3, 4 and improved band 6). Classification accuracy increased when we used improved thermal data instead of original thermal data, which means there was improvement in feature identification.

In the present study, classification for all combinations of data was done using ANN and MLH methods and the results are tabulated (Table 1). These combinations include

1) Bands 2, 3, 4 and original Daytime thermal using ANN and MLH
2) Bands 2, 3, 4 and Improved Daytime TIR Data using 3 bands as input (IDTI3) using ANN and MLH

3) Bands 2, 3, 4 and Improved Daytime TIR Data using 4 bands as input (IDTI4) using ANN and MLH

4) Bands 2, 3 and 4 using ANN and MLH

5) Bands 2, 3, 4, original Daytime and Nighttime thermal using ANN and MLH

6) Bands 2, 3, 4, IDTI3 and Improved Nighttime TIR Data using 3 bands as input (INTI3) using ANN and MLH

7) Bands 2, 3, 4, IDTI4 and Improved Nighttime TIR Data using 4 bands as input (INTI4) using ANN and MLH

Table 1: Classification Accuracies for each combination of data

\begin{tabular}{|c|c|c|}
\hline Method & ANN & MLH \\
\hline $\begin{array}{c}\text { With bands 2,3 and 4 } \\
\text { With bands2, 3,4 and } \\
\text { raw day TIR }\end{array}$ & $76.25 \%$ & $76.17 \%$ \\
\hline $\begin{array}{c}\text { With bands2, 3,4 and } \\
\text { IDTI3 }\end{array}$ & $90.57 \%$ & $87.85 \%$ \\
\hline $\begin{array}{c}\text { With bands2, 3,4 and } \\
\text { IDTI4 }\end{array}$ & $95.57 \%$ & $83.28 \%$ \\
\hline $\begin{array}{c}\text { With bands2, 3, 4 } \\
\text { and raw day \& night } \\
\text { TIR }\end{array}$ & $81.85 \%$ & $82.85 \%$ \\
\hline $\begin{array}{c}\text { With bands2, 3,4 and } \\
\text { IDTI3 \& INTI3 }\end{array}$ & $68 \%$ & $83.29 \%$ \\
\hline $\begin{array}{c}\text { With bands2, 3,4 and } \\
\text { IDTI4 \& INTI4 }\end{array}$ & $85.42 \%$ & $84.43 \%$ \\
\hline
\end{tabular}

\section{COMPARISONS AND DISCUSSION}

Classification accuracy improved when raw daytime thermal data were used in addition to MS data by ANN. When only MS data were used in classification it resulted only $76.2 \%$ accuracy. In the classified image with MS data thick vegetation, light vegetation, marshy land and open ground (barren land) were misclassified. When raw daytime TIR data were also used with MS data, accuracy increased to $90.57 \%$ (Fig 1). In the classified image with MS data and raw daytime TIR data only few pixels of barren land and marshy land were misclassified. There was further improvement in accuracy when improved daytime thermal data with 4 bands (IDTI4) were used in classification by ANN instead of raw daytime thermal data along with MS data. In the classified image with MS data and IDTI4 all the classes were classified accurately. Each and every class had an individual accuracy of more than 92\% accuracy. Overall accuracy was 95.57\% (Fig 2). 

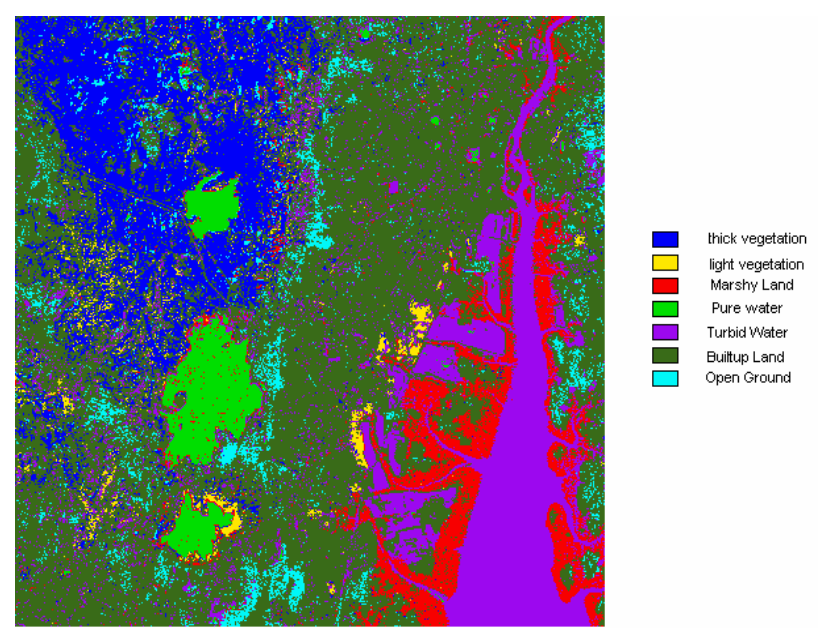

Figure 1: ANN Classified image using bands 2, 3, 4 and original Daytime TIR data

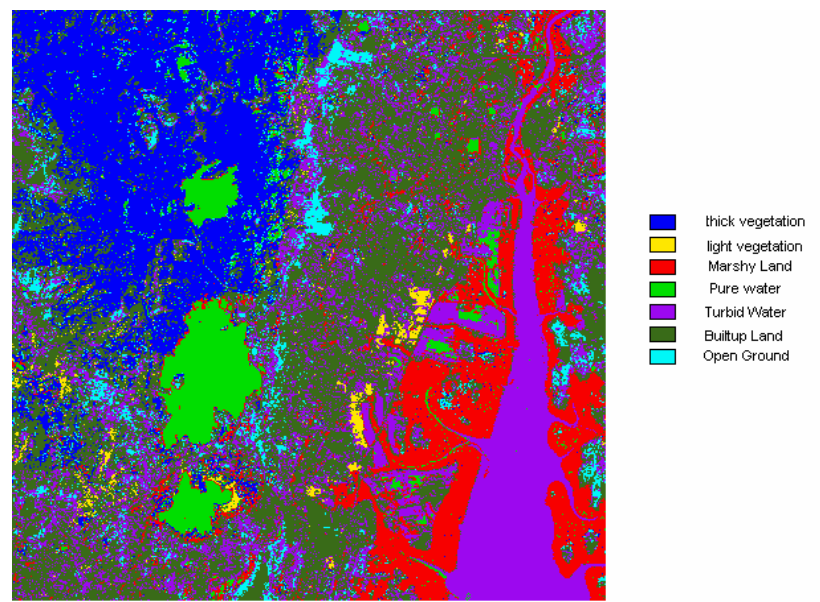

Figure 2: ANN Classified image using bands 2, 3, 4 and IDTI4

Classification accuracy decreased when daytime improved image with 3 bands (IDTI4) by ANN was used instead of daytime raw thermal data. In the classified image with MS data and IDTI3 all the thick vegetation and light vegetation was misclassified as built-up land. Hence it gave the lowest accuracy of $69 \%$. This gives the fact that improved image by ANN with 4bands (IDTI4) is better than improved image with 3 bands (IDTI3). When nighttime data were used along with MS data and daytime thermal data there was a slight reduction in classification accuracy to $81.85 \%$. This may be due to a small error in registration between day and nighttime data. In the classified image with MS data, raw daytime and nighttime TIR data some pixels of thick vegetation and barren land were misclassified as built-up land. In the classified image with MS data, IDTI3 and INTI3 thick vegetation, barren land and light vegetation were misclassified as built-up land. It gave an accuracy of $68 \%$. Among the classified images where nighttime data were used, maximum accuracy was obtained when MS data, IDTI4 and INTI4 were used. It gave an accuracy of $85.42 \%$. Classification was also done for all the above combinations of data using MLH. In all the cases ANN proved to be a better method than MLH. In classification with MLH also, there was always an increase in accuracy when improved daytime thermal data (IDTI4) were used instead of raw daytime thermal data. Classification accuracy was always higher when daytime thermal data were used along with MS data. But there was a slight reduction in classification accuracy when nighttime thermal data were included. The reasons for this may be small a error in registration between daytime and nighttime data and also there may be higher correlation between day and nighttime data.

\section{CONCLUSIONS}

The main objectives of the present study include effects of improved daytime and nighttime TIR data in classification. Details present in the TIR data are very useful in classification. Though thermal data was available along with multispectral data, it was not used because of its very coarse spatial resolution. Using our new method not only one can bring the thermal data to a resolution of the multispectral data but also retain the thermal properties and patterns. Hence using this method we now have an opportunity to utilize thermal data as an extra band for land use/land cover classification.

The results indicated enormous improvement in classification accuracy when thermal data were used along with multi spectral data. Classification accuracy was much better when improved thermal data were used instead of raw thermal data. There was no increase in classification accuracy when nighttime data were used along with daytime thermal and MS data compared to the results obtained with the MS data and daytime TIR data. Linear features such as roads, railway networks etc were easily identified when the improved TIR data were used along with visible and near infrared data in multispectral image classification. With out disturbing the thermal properties of the data we could use it for land use/land cover map preparation. Future work could include image classification using advanced methods like Neuro-Fuzzy algorithms.

\section{REFERENCES}

1. Stephen M. Leak And Venugopal, G. 'Thematic Mapper Thermal Infrared Data in Discriminating Selected Urban Features'. International Journal of Remote Sensing, Vol-11, No.5, pp. 841-857, 1990.

2. Sabins F. Floyd 'Remote Sensing Principles and Interpretation', Third Edition. W. H. Freeman and Company, New York, 1997. 
3. Price C. John 'The Contribution of Thermal Data in Landsat Multi Spectral Classification'. Photogrammetric Engineering and Remote Sensing, Vol-47, No-2, pp. 229-236, 1981.

4. Ormsbay P. James, 'The Use of Landsat-3 Thermal Data to Help Differentiate Land Covers'. Remote sensing of environment, vol-12, pp. 92-105, 1982.

5. Heermann, P. D., and Khazenie, K., 'Classification of Multispectral Remote Sensing Data using a Back Propagation Neural Network', IEEE Transactions on Geoscience and Remote Sensing, Vol. 30, No.1, pp.81-88, 1992.

6. Bischof, H., Schneider, W., and Pinz, A.J., 'Multispectral Classification of Landsat-Images Using NEURAL Networks', IEEE Transactions on Geoscience and Remote Sensing, Vol. 30, No.3, pp.483-490, 1992.

7. Paola, J.D., and Robert, A.S., 'A detailed comparison of back-propagation neural network and maximum likelihood classifiers for urban land use classification', IEEE Transactions on Geoscience and remote sensing, vol.33, no.4, pp 981-996, 1995.

8. Arora, M.K., and Foody, G.M., 'Log linear modeling for the evaluation of the variables affecting the accuracy of probabilistic, fuzzy and neural network classifications', International Journal of Remote sensing, Vol.18, N0.4, pp.785798, 1997.

9. Foody, G.M., and Arora, M.K., 'An Evaluation of some factors affecting the accuracy of classification by an Artificial Neural Network', International Journal of Remote sensing, Vol.18, N0.4, pp.799-810, 1997

10. Benediktsson, J.A., and Sveinson, J.R., 'Feature extraction for multisource data classification with Artificial Neural networks', International Journal of Remote sensing, Vol.18, N0.4, pp.727-740, 1997.

Chavez S Pat, Jr., Stuart C. Sides, and Labrey A. Anderson 'Comparison of Three Different Methods to Merge Multi Resolution and Multi Spectral Data: Landsat TM Data and SPOT Panchromatic.

Photogrammetric Engineering and Remote Sensing, Vol-57, No-3, pp. 295-303, 1991.

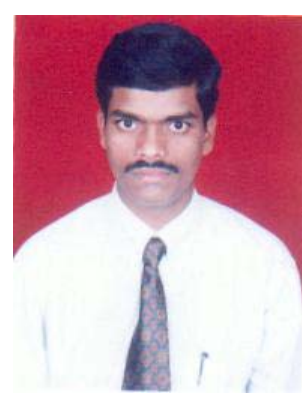

Venkateswarlu Cheruku graduated with a Bachelor's degree in Civil Engineering from KITS Warangal, India. He earned his Master's in Remote Sensing from Indian Institute of Technology, Mumbai, India. He is presently working as a software engineer at Infosys Technologies Ltd. Hyderabad,
India. His interests include Digital Image Processing Techniques in Thermal Infrared Data, Temperature Mapping Using Thermal Data, Artificial Neural Networks and Fuzzy Classification Techniques.

Sumanth Yenduri was born in 1976, Rajahmundry, India. He graduated with a Bachelor's degree in Computer Science and Systems Engineering from Andhra University, India in 1999. He earned his Master's degree in Systems Science from Louisiana State University in 2002.

Presently he is working towards his doctoral degree in the Computer Science Department at Louisiana State University. His research interests are in the areas of Image Classification, Software Coding Standards, and Programming Languages.

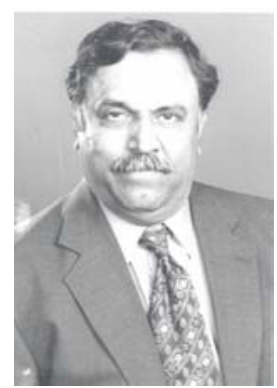

Dr. S. S. lyengar is the Chairman and Roy Paul Daniels Chaired Professor of Computer Science and is also Satish Dhawan Chaired Professor. He is the Distinguished Research Master Award winning Professor of the Computer Science Department at Louisiana State University. He has been involved with research in high-performance algorithms, data structures, sensor fusion, data mining, and intelligent systems since receiving his Ph.D. degree (in 1974 at Mississippi State University) and his M.S. from the Indian Institute of Science (1970). He has directed over 30 Ph.D. candidates, many of whom are faculty at major universities worldwide or scientists or engineers at national labs/industry around the world. $\mathrm{He}$ has served as a principal investigator on research projects supported by the Office of Naval Research (ONR), Defense Advanced Research Project Agency (DARPA), the National Aeronautics and Space Administration (NASA), the National Science Foundation (NSF), California Institute of Technology's Jet Propulsion Laboratory (JPL), the Department of Navy-NORDA, the Department of Energy (DOE), LEQSF-Board of Regents, and the U.S. Army Research Office. His publications include 13 books (authored or coauthored textbooks; Prentice-Hall, CRC Press, IEEE Computer Society Press, John Wiley \& Sons, etc.) and over 280 research papers in refereed journals and conference in areas of high-performance parallel and distributed algorithms and data structures for image processing and pattern recognition, and distributed data mining algorithms for biological databases. His books have been used in Berkeley, Purdue, University of Southern California, University of New Mexico, etc. He was a visiting professor at the Jet Propulsion Laboratory-Cal. Tech, Oak Ridge National Laboratory, the Indian Institute of Science, and at the University of Paris. Dr. lyengar has served as 
an associate editor for the Institute of Electrical and Electronics Engineers and as guest editor for the IEEE Transactions on Knowledge and Data Engineering, IEEE Transactions and SMC, IEEE Transactions on Software Engineering, Journal of Theoretical Computer Science, Journal of Computer and Electrical Engineering, Journal of the Franklin Institute, Journal of American Society of Information Science, International Journal of High Performance Computing Applications, etc. He has served on review panel committees for NSF, NASA, DOEORNL, the U.S. Army Research Office, etc. He has been on the prestigious National Institute of HealthNLM Review Committee, in the area of Medical Informatics for 4 years. Dr. lyengar is a series editor for Neuro-Computing of Complex Systems for CRC Press.

In 1998 Dr. lyengar was the winner of the IEEE Computer Society Technical Achievement Award for Outstanding Contributions to Data Structures and Algorithms in Image Processing and Sensor Fusion Problems. This is the most prestigious research award from IEEE Computer Society. Dr. lyengar was awarded the LSU Distinguished Faculty Award for Excellence in Research, the Hub Cotton Award for Faculty Excellence, and the LSU Tiger Athletic Foundation Teaching Award in 1996. He has been a consultant to several industrial and government organizations (JPL, NASA etc.). In 1999, Professor lyengar won the most prestigious research award titled Distinguished Research Award and a university medal for his research contributions in optimal algorithms for sensor fusion/image processing.

He is also a Fellow of Association of Computing Machinery (ACM), a Fellow of the IEEE, a Fellow of American Association of Advancement of Science (AAAS), a Williams Evans Fellow, IEEE Distinguished Visitor, etc. He has been a Distinguished Lecturer for the IEEE Computer Society for the years 1995 - 1998, an ACM National Lecturer (1986-1992), and Member of the Distinguished SIAM Lecturer Program (2000-2002). He was a member of the ACM accreditation committee for 2000 - 2002. He is a member of the New York Academy of Sciences. He has been the Program Chairman for many national/international conferences. He has given over 50 plenary talks and keynote lectures at numerous national and international conferences. 